\title{
Ocular Perfusion Pressure and Severity of Glaucoma: Is There
} a Link?

\author{
Ch'ng TW' ${ }^{1}$, Chua $\mathrm{CY}^{2}$, Ummi Kalsom MA ${ }^{3}$, Azhany $\mathrm{Y}^{4}$, Gong $\mathrm{VHM}^{5}$, Rasool $\mathrm{AHG}^{6}$, Liza-Sharmini $\mathrm{AT}^{7}$
}

\begin{abstract}
Aim and objective: To establish the association between 12-month inter-visits ocular perfusion pressure (OPP) and severity of glaucoma. Materials and methods: A total of 164 eyes ( 60 primary open-angle glaucoma, 52 normal-tension glaucoma, and 52 primary angle-closure glaucoma) of 164 patients were analyzed in this prospective cohort study. Patients who demonstrated good compliance to treatment were recruited. The severity of glaucoma was stratified according to the Advanced Glaucoma Intervention Study (AGIS) score based on reliable and reproducible visual field analysis at baseline. OPP was obtained at baseline and a 3-monthly follow-up visit over a 12-month duration. The pattern and fluctuation of mean OPP were studied. Repetitive measure ANOVA and one-way ANOVA were used for statistical analysis.

Results: The inter-visits mean OPP $(p=0.010)$, systolic $\operatorname{OPP}(p=0.020)$, diastolic $\operatorname{OPP}(p=0.010)$, systolic blood pressure $(p=0.040)$, and diastolic blood pressure $(p=0.006)$ showed significant difference with severity of glaucoma. There was no significant difference between mean intervisits intraocular pressure (IOP) and severity of glaucoma $(p=0.410)$. The end-stage glaucoma group had the lowest mean OPP and widest inter-visit mean OPP fluctuation. Early and mild glaucoma patients demonstrated higher mean OPP compared with moderate, severe, and end-stage glaucoma.

Conclusion: There is a significant association between OPP and the severity of glaucoma. Balancing blood pressure and IOP is important in optimizing adequate perfusion and prevent further damage to the optic nerve head.

Keywords: Blood pressure, Intraocular pressure, Ocular perfusion pressure, Severity of glaucoma.

Journal of Current Glaucoma Practice (2021): 10.5005/jp-journals-10078-1305
\end{abstract}

\section{INTRODUCTION}

Intraocular pressure (IOP) is a known modifiable risk factor for glaucoma. Thus, the objective of glaucoma management is to lower the IOP to achieve a safe target pressure. However, glaucomatous damage still occurs in some patients despite a significant reduction of IOP. This implies that other factors may be responsible. About $20 \%$ of normal-tension glaucoma (NTG) patients in The Collaborative Normal-Tension Glaucoma (CNTG) study progressed despite achieving 30\% IOP reduction from baseline.

Low ocular perfusion pressure (OPP) is also identified as a potential risk factor for the progression of glaucoma. The EgnaNeumarkt study, Proyecto VER, and the Baltimore Eye Survey reported that low diastolic OPP (dOPP) was associated with three-, four-, and six-folds increase risk of open-angle glaucoma (OAG), respectively. ${ }^{2-4}$ The Barbados Eye Study and the Los Angeles Latino Eye Study also showed that mean OPP (mOPP) and systolic OPP (sOPP) were associated with glaucoma. ${ }^{5,6}$ Ocular perfusion pressure affects ocular blood flow to the optic nerve head $(\mathrm{ONH}) .^{7-9}$

Circulatory abnormalities by occlusion of small vessels produce infarction of the ONH. ${ }^{10}$ Persistently high IOP mechanically causes stretching of the laminar beams, which subsequently damages the retinal ganglion cell axons. ${ }^{11,12}$ Ocular blood flow reduces with decreasing perfusion pressure, especially when vascular autoregulation is impaired. ${ }^{7-9}$ Autoregulatory mechanism maintains the blood flow to the ONH to a certain extent. Thus, theoretically, the combination of high IOP and low BP may lead to the progression of glaucoma.

In addition, diurnal OPP fluctuation is also a risk factor for the progression of glaucoma. ${ }^{13}$ Long-standing instability of OPP may also be responsible for chronic optic disk circulation insufficiency
${ }^{1}$ Department of Ophthalmology, School of Medical Science, Universiti Sains Malaysia, Health Campus, Kubang Kerian, Kelantan, Malaysia; Department of Ophthalmology, Hospital Raja Permaisuri Bainun, Ipoh, Perak, Malaysia

${ }^{2}$ Department of Ophthalmology, Hospital Bukit Mertajam, Bukit Mertajam, Penang, Malaysia

3,5Department of Ophthalmology, Hospital Raja Permaisuri Bainun, Ipoh, Perak, Malaysia

4,7Department of Ophthalmology, School of Medical Science, Universiti Sains Malaysia, Health Campus, Kubang Kerian, Kelantan, Malaysia

${ }^{6}$ Department of Pharmacology, School of Medical Sciences, Universiti Sains Malaysia, Health Campus, Kubang Kerian, Kelantan, Malaysia

Corresponding Author: Liza-Sharmini A Tajudin, Department of Ophthalmology, School of Medical Science, Universiti Sains Malaysia, Health Campus, Kubang Kerian, Kelantan, Malaysia, Phone: +60199179227, e-mail: liza@usm.my; sharminiliz@live.com

How to cite this article: Ch'ng TW, Chua CY, Ali UKM, et al. Ocular Perfusion Pressure and Severity of Glaucoma: Is There a Link? J Curr Glaucoma Pract 2021;15(2):78-85.

Source of support: This study is supported by the Research University Grant Universiti Sains Malaysia 1001/PPSP/812101.

Conflict of interest: None

that ultimately causes further deterioration and progression of glaucoma. ${ }^{14}$ Asrani et al. found that diurnal IOP and short-term IOP fluctuations over the days are important predictors for the progression of glaucoma. ${ }^{15}$

() Jaypee Brothers Medical Publishers. 2021 Open Access This article is distributed under the terms of the Creative Commons Attribution 4.0 International License (https://creativecommons.org/licenses/by-nc/4.0/), which permits unrestricted use, distribution, and non-commercial reproduction in any medium, provided you give appropriate credit to the original author(s) and the source, provide a link to the Creative Commons license, and indicate if changes were made. The Creative Commons Public Domain Dedication waiver (http://creativecommons.org/publicdomain/zero/1.0/) applies to the data made available in this article, unless otherwise stated. 
However, 24-hour monitoring of OPP is impractical for most patients and ophthalmologists. It is usually conducted in the sleep lab or requires hospital admission that disturbs the patient's daily routine. The hospital setting and sleep lab create an artificial environment. The 24-hour blood pressure (BP) measurement performed may not truly reflect a patient's daily rhythm. ${ }^{16}$ Intervisit OPP is, therefore, more practical in clinical practice with less disruption to the patient's routine and cost-effective. The inter-visit OPP is best conducted according to the patient's routine clinic appointment. The relationship between OPP and the severity of glaucoma has not been studied thoroughly. This study aimed to study the association between 12 months inter-visits OPP with different types of primary glaucoma. The inter-relationship between $\mathrm{OPP}, \mathrm{BP}, \mathrm{IOP}$, and primary glaucoma was also explored.

\section{Materials and Methods}

A prospective cohort study was conducted involving patients with primary open-angle glaucoma (POAG), normal-tension glaucoma (NTG), and primary angle-closure glaucoma (PACG), who were seen in the eye clinics of Hospital Raja Permaisuri Bainun (HRPB), Perak and Hospital Universiti Sains Malaysia (HUSM), Kelantan, Malaysia, between September 2010 and September 2012 to establish the association between 12-month inter-visits OPP and severity of glaucoma. This study received ethical approval from the Research Ethics Committee, Ministry of Health Malaysia (NMRR-101244-7957) and the Research Ethics Committee, Universiti Sains Malaysia (reference no. USMKK/PPP/JEPeM[234.3(12)]). This study was conducted in accordance with the Declaration of Helsinki for human research.

\section{Patients Recruitment}

In this study, POAG was diagnosed when there was the presence of structural and/or functional changes of glaucoma with IOP. This was based on the definition by the International Society Geographical and Epidemiological Ophthalmology (ISGEO). ${ }^{17}$ NTG was based on the presence of structural and functional changes of glaucoma without any secondary causes, open angles on gonioscopy, and median IOP of $20 \mathrm{~mm} \mathrm{Hg}$ or less on 10 baseline measurements. ${ }^{18}$ PACG was defined as occludable drainage angle of the eye with features that suggest trabecular obstruction by the peripheral iris (peripheral anterior synechiae formation, increased IOP, iris whirling, and glaukomflecken) with the glaucomatous optic disk. ${ }^{17}$

We recruited patients with primary glaucoma (POAG, NTG and PACG) who were treated with topical pressure-lowering medications without any history of filtrating surgery. They demonstrated good adherence and persistence to treatment. Their selection was through non-probability sampling. Patients who were diagnosed with secondary glaucoma (angle recession glaucoma, pseudoexfoliation glaucoma, and steroid-induced glaucoma) were excluded from this study. Those with concurrent ocular disease such as retinitis pigmentosa, refractive error $-3.0 \mathrm{D}$ or more, narrow-angle without glaucomatous disk changes were excluded. Those with a history of glaucoma surgery or other previous ocular surgery except pterygium and cataract surgery were also excluded. To ensure a good quality of visual field assessment, patients with vision worse than 6/60, media opacity such as dense cataract, corneal scar, vitreous hemorrhage, post panretinal photocoagulation (PRP), tilted disk, and neuro-ophthalmic conditions were excluded. In addition, those who required surgical (glaucoma filtration surgery) intervention during follow-up visits and intravitreal anti-vascular endothelial growth factor (anti-VEGF) injection were also excluded from this study. The right eye was selected regardless of the severity of glaucoma if both eyes were eligible.

\section{Clinical Procedures and Assessment of Glaucoma Severity}

At each visit, the participants were examined with a slit lamp and best corrected visual acuity (BCVA) was recorded. In sitting position, Goldmann Applanation tonometer (Haag-Streit, Bern, Switzerland) was used to take two IOP measurements between 8 am and 12 noon. The third IOP reading was taken if the first two measurements differed $>2 \mathrm{~mm} \mathrm{Hg}$. Angle assessment was done with a Goldmann two-mirror gonio-lens (Ocular Instruments, Bellevue, Washington, USA). By using Pachymeter (Topcon Specular Microscope SP2000P, Tokyo Japan), central corneal thickness (CCT) was measured. The two latest reproducible and reliable consecutive visual fields were obtained from the hospital medical information system. In cases, when there was unavailable of the latest reliable and reproducible visual fields, two consecutive visual fields were then conducted within 3 months post-recruitment period. The visual field test was performed using Humphrey Field Analyser 750i (Carl Zeiss Meditec Inc., USA) according to the Swedish Interactive Threshold Algorithm Standard (SITA-Standard) with 24-2 full threshold strategy.

The severity of glaucoma was categorized according to the Advanced Glaucoma Intervention Study (AGIS) score based on two consecutive, reproducible, and reliable Humphrey visual field 24-2 analyzes. A visual field is considered reliable when a falsenegative response of $33 \%$ or less, fixation loses not $>20 \%$, and false-positive response $<33 \%$. The first scoring was done by the primary investigator (CTW) and the re-scoring was done by the fellowship-trained glaucoma consultant (LSAT). The severity was determined by the agreement of two assessors.

\section{Other Measurements}

Upon arrival to the eye clinic, patients with primary glaucoma were allowed to rest before brachial BP measurement. Brachial BP was measured using a sphygmomanometer (Accoson Dekamet, England) and stethoscope (Littmann, England) in the sitting position by the primary investigator (CTW) according to the protocol recommended by the Multi-ethnic Study of Atherosclerosis. ${ }^{19}$ Two readings were obtained with 5 minutes rest in-between to ensure the accuracy of the BP reading. The final BP was based on the mean pressure of these two readings to eliminate bias. If systolic blood pressure (sBP) differs $>10 \mathrm{~mm} \mathrm{Hg}$ or diastolic blood pressure (dBP) differs $>5 \mathrm{~mm} \mathrm{Hg}$, a third measurement was taken. Intraocular pressure and BP measurements were taken at almost the same time point.

Fasting blood sugar (FBS), fasting lipid profile (FLP), body mass index (BMI), and waist circumference were obtained from the medical record in cases of known hyperlipidemia and diabetes mellitus. In subjects without evidence or those not known to have hyperlipidemia or diabetes mellitus, FBS or FLP was obtained on their next scheduled follow-up.

\section{Ocular Perfusion Pressure}

OPP is the difference of pressure between systemic BP and IOP. OPP is further divided into systolic ocular perfusion pressure (sOPP) and diastolic ocular perfusion pressure (dOPP). SOPP is the difference of pressure between systolic blood pressure (sBP) and IOP, calculated using formula sOPP = sBP-IOP. dOPP is the difference of pressure between diastolic blood pressure (dBP) and IOP, given by the 
formula dOPP $=\mathrm{dBP}-\mathrm{IOP}$. Mean OPP is the difference of pressure between mean blood pressure (MBP) and IOP calculated using the formula $\mathrm{mOPP}=2 / 3 \mathrm{mBP}-\mathrm{IOP}^{9,20}$ Ocular perfusion pressure was obtained at baseline and at 3 monthly follow-up visits in a year. The magnitude of difference between the highest mean OPP and lowest mean OPP is fluctuation of ocular perfusion pressure (fOPP).

\section{Statistical Analysis}

One-way ANOVA was used to compare the difference of numerical data such as mean age, mean IOP, mean BP according to the type of glaucoma. Pearson Chi-square test and Fisher's exact test were used for categorical data. The association between 12 monthly intervisits OPP and severity of glaucoma was analyzed using Repeated Measures Analysis of Variance (RM-ANOVA). Multiple paired $t$-tests with Bonferroni correction were also conducted. $p$ value $<0.05$ is considered statistically significant.

\section{Results}

\section{Demographic Data}

A total of 208 patients (208 eyes) were recruited but only 164 eyes of 164 glaucoma patients were included for the final analysis. Forty-four patients failed to complete the 12-month follow-up. There were almost equal numbers of participants in POAG, NTG, and PACG ( 60 eyes, 52 eyes, and 52 eyes, respectively). All recruited patients were of Asian descent and the distribution of ethnicity was almost similar to the Malaysian demographics; Malays 57.9\%, Chinese 38.4\%, and Indians 3.7\%. Hypertension was the commonest systemic comorbidity (Table 1).
Among the groups, there were significant differences in duration of follow-up (longest in NTG, followed by POAG and PACG), the number of male participants (more in POAG and NTG group), diabetics (almost double in POAG compared the other groups), BMI, and waist circumference (highest in PACG, followed by NTG and POAG group) (Table 2).

Based on the modified AGIS score, there were $16.5 \%$ early, $39.6 \%$ mild, $28.0 \%$ moderate, $11.6 \%$ severe, and $4.3 \%$ end-stage glaucoma patients. The majority of our patients were graded to have mild to moderate glaucoma (67.6\%). However, there was no statistically significant difference according to the severity of glaucoma (Table 2).

\section{OPP and Severity of Glaucoma}

Figure $1 \mathrm{~A}$ shows a significant association between mOPP and severity of AGIS score. Overall, early and mild glaucoma groups had higher mOPP as compared with moderate, severe, and end-stage groups ( $p=0.010)$. The end-stage group had the widest inter-visit mOPP fluctuation (Fig. 1).

We also observed a significant difference between OPP subcategories (sOPP, $p=0.020$ and dOPP, $p=0.010$ ) and severity of glaucoma (Figs $1 \mathrm{~B}$ and $\mathrm{C}$ ). End-stage glaucoma patients had consistently the lowest sOPP and a V-shaped pattern of dOPP. Both sOPP and dOPP dipped to the lowest mean at 6 months. On the contrary, minimal fluctuation was seen in the early and mild glaucoma group and both were found to have higher mean sOPP and dOPP. There was no statistically significant difference in the mOPP according to the time of visits between POAG, NTG, and PACG groups except at 3 months (Fig. 1).

Table 1: Demographic and clinical characteristics according to the severity of glaucoma

\begin{tabular}{|c|c|c|c|c|c|}
\hline & Early $(n=27)$ & Mild $(n=65)$ & Moderate $(n=46)$ & Severe $(n=19)$ & End-stage $(n=7)$ \\
\hline $\begin{array}{l}\text { Mean age (at first diagno- } \\
\text { sis) (mean, SD) }\end{array}$ & $56.9(8.0)$ & $60.4(8.9)$ & $63.8(9.9)$ & $67.1(9.2)$ & $65.1(8.5)$ \\
\hline \multicolumn{6}{|l|}{ Type of glaucoma $(n, \%)$} \\
\hline POAG & $12(44.4)$ & $23(35.4)$ & $16(34.8)$ & $7(36.8)$ & $2(28.6)$ \\
\hline NTG & $7(26.0)$ & $24(36.9)$ & $15(32.6)$ & $5(26.4)$ & $1(14.3)$ \\
\hline PACG & $8(29.6)$ & $18(27.7)$ & $15(32.6)$ & $7(36.8)$ & $4(57.1)$ \\
\hline \multicolumn{6}{|l|}{ Systemic disease } \\
\hline $\operatorname{HTN}(n, \%)$ & $20(74.1)$ & $44(67.7)$ & $31(67.4)$ & $11(57.9)$ & $5(71.4)$ \\
\hline $\operatorname{DM}(n, \%)$ & $12(44.4)$ & $15(23.1)$ & $18(39.1)$ & $4(21.1)$ & $3(42.9)$ \\
\hline $\operatorname{IHD}(n, \%)$ & $3(11.1)$ & $15(23.1)$ & $8(17.4)$ & $1(5.3)$ & $1(14.3)$ \\
\hline $\operatorname{HPL}(n, \%)$ & $15(55.6)$ & $19(29.2)$ & $20(43.5)$ & $6(31.6)$ & $3(42.9)$ \\
\hline \multirow[t]{2}{*}{ CCT, $\mu$ m (mean, SD) } & 519.0 & 522.7 & 515.7 & 516.8 & 515.1 \\
\hline & $(26.0)$ & $(29.0)$ & $(63.5)$ & $(24.8)$ & $(25.9)$ \\
\hline BMI (mean, SD) & $24.7(4.6)$ & $24.4(5.2)$ & $24.7(3.3)$ & $24.1(5.1)$ & $23.8(3.0)$ \\
\hline $\begin{array}{l}\text { Waist circumference, cm } \\
\text { (mean, SD) }\end{array}$ & $93.8(8.4)$ & $94.5(12.7)$ & $90.8(10.5)$ & $93.5(11.5)$ & 90.7 (7.6) \\
\hline \multicolumn{6}{|l|}{$\operatorname{Sex}(n, \%)$} \\
\hline Male & $14(51.9)$ & $43(66.2)$ & $22(47.8)$ & 7 (36.8) & $6(85.7)$ \\
\hline Female & $13(48.1)$ & $22(33.8)$ & $24(52.2)$ & $12(63.2)$ & $1(14.3)$ \\
\hline \multicolumn{6}{|l|}{ Ethnicity $(n, \%)$} \\
\hline Malay & 15 (55.6) & $38(58.5)$ & $24(52.2)$ & $12(63.2)$ & $6(85.7)$ \\
\hline Chinese & $12(44.4)$ & 24 (36.9) & $20(43.5)$ & 6 (31.6) & $1(14.3)$ \\
\hline Indian & 0 & $3(4.6)$ & $2(4.3)$ & $1(5.3)$ & 0 \\
\hline
\end{tabular}

POAG, primary open-angle glaucoma; NTG: normal-tension glaucoma; PACG, primary angle-closure glaucoma; HTN, hypertension; DM, diabetes mellitus; IHD, ischemic heart diseases; HPL, hyperlipidemia; CCT, central corneal thickness; BMI, body mass index 
Table 2: Comparison of demographic data and clinical characteristics between POAG, NTG, and PACG patients

\begin{tabular}{|c|c|c|c|c|}
\hline Demographic characteristic & POAG $(N=60)$ & NTG $(N=52)$ & PACG $(N=52)$ & pvalue \\
\hline Mean age (at first diagnosis) (mean, SD) & $63.0(9.4)$ & $59.6(10.3)$ & $62.3(8.5)$ & $0.148^{@}$ \\
\hline Mean age (at recruitment) (mean, SD) & $68.2(9.4)$ & $65.6(8.8)$ & $66.2(8.7)$ & $0.269^{@}$ \\
\hline Duration of follow-up (mean, SD) & $5.4(4.4)$ & $6.2(4.4)$ & $4.2(3.3)$ & $\mathbf{0 . 0 3 7 ^ { @ }}$ \\
\hline \multicolumn{5}{|l|}{$\operatorname{Sex}(n, \%)$} \\
\hline Male & $43(71.7)$ & $30(57.7)$ & $19(36.5)$ & \multirow[t]{2}{*}{$0.001^{\#}$} \\
\hline Female & $17(28.3)$ & $22(42.3)$ & $33(63.5)$ & \\
\hline \multicolumn{5}{|l|}{ Ethnicity $(n, \%)$} \\
\hline Malay & $30(50.0)$ & $32(61.6)$ & $33(63.5)$ & \multirow[t]{3}{*}{$0.597^{*}$} \\
\hline Chinese & $28(46.7)$ & $18(34.6)$ & $17(32.7)$ & \\
\hline Indian & $2(3.3)$ & $2(3.8)$ & $2(3.8)$ & \\
\hline Type of glaucoma $(n, \%)$ & $60(36.6)$ & $52(31.7)$ & $52(31.7)$ & \\
\hline Central cornea thickness, $\mu \mathrm{m}$ (mean, SD) & $527.6(28.1)$ & $515.6(31.0)$ & $512.8(40.7)$ & $0.118^{@}$ \\
\hline \multicolumn{5}{|l|}{ Systemic disease } \\
\hline Hypertension ( $n, \%)$ & $44(73.3)$ & $38(73.1)$ & $29(55.8)$ & $0.085^{\#}$ \\
\hline Diabetes mellitus ( $n, \%)$ & $27(45.0)$ & $10(19.2)$ & $15(28.8)$ & $0.012^{\#}$ \\
\hline Ischemic heart disease $(n, \%)$ & $14(23.3)$ & $8(15.4)$ & $6(11.5)$ & $0.236^{\#}$ \\
\hline Hyperlipedemia ( $n, \%)$ & $22(36.7)$ & $23(44.2)$ & $18(34.6)$ & $0.566^{\#}$ \\
\hline BMI (mean, SD) & $23.1(3.5)$ & $24.8(5.5)$ & $25.8(4.0)$ & $0.004^{@}$ \\
\hline Waist circumference (mean, SD) & $89.6(11.1)$ & $94.6(11.2)$ & $95.6(10.2)$ & $0.008^{@}$ \\
\hline Neck circumference (mean, SD) & $37.4(8.3)$ & $35.9(4.1)$ & $36.4(6.0)$ & $0.454^{@}$ \\
\hline \multicolumn{5}{|l|}{$\operatorname{APAC}(n, \%)$} \\
\hline No & - & - & $35(67.3)$ & \multirow[t]{2}{*}{-} \\
\hline Yes & - & - & $17(32.7)$ & \\
\hline \multicolumn{5}{|l|}{ AGIS score $(n, \%)$} \\
\hline Early & $12(20.0)$ & 7 (13.9) & $8(15.4)$ & \multirow[t]{5}{*}{$0.828^{*}$} \\
\hline Mild & $23(38.3)$ & $24(46.1)$ & $18(34.6)$ & \\
\hline Moderate & $16(26.7)$ & 15 (28.7) & $15(28.8)$ & \\
\hline Severe & $7(11.7)$ & $5(9.5)$ & $7(13.5)$ & \\
\hline End-stage & $2(3.3)$ & $1(1.8)$ & $4(7.7)$ & \\
\hline
\end{tabular}

BMI, body mass index; APAC, acute presentation of angle-closure; AGIS, advanced glaucoma intervention study

$\# p<0.05$ is considered statistical significance based on Pearson's Chi-square

${ }^{*} p<0.05$ is considered statistical significance based on Fisher's exact test

${ }^{\circledR} p<0.05$ is considered statistical significance based on one-way ANOVA

\section{IOP and Severity of Glaucoma}

We found no significant difference between mean IOP and different severity of glaucoma ( $p=0.410$ ) (Fig. 2). However, the end-stage glaucoma group was observed to have the highest mean IOP and the widest mean IOP fluctuation. This is in contrast with the mild glaucoma group which demonstrated the lowest mean IOP and the least mean IOP fluctuation. Interestingly, the early glaucoma group showed a "see-saw" pattern of mean IOP but the value maintained $<18 \mathrm{~mm} \mathrm{Hg}$.

\section{BP and Severity of Glaucoma}

Figure $3 \mathrm{~A}$ shows a statistically significant association between mean inter-visit sBP over 12 months period and severity of glaucoma ( $p=0.040)$. End-stage glaucoma consistently had the lowest sBP among the groups, dipped to lowest at 9 months before picking up at 12 months. The severe glaucoma group had the highest SBP at the beginning but showed a steady decline at subsequent follow-up visits.

Similarly, mean dBP was also found to have a statistically significant difference with the severity of glaucoma $(p=0.006)$
(Fig. 3B). The early and mild glaucoma group was observed to have higher mean $\mathrm{dBP}$ when compared with the moderate to severe glaucoma group. The end-stage glaucoma group was noted to have the widest mean dBP fluctuation, with a sudden decrease at 3 and 6 months, followed by a gradual increase at 9 months.

\section{Discussion}

The elusive role of OPP in glaucoma remains the interest of many researchers till now. Ocular perfusion pressure is calculated mathematical according to the assumption that IOP represents the venous pressure and $\mathrm{BP}$ as the arterial pressure. The association between inter-visit mOPPs and the severity of glaucoma was statistically significant. Our end-stage glaucoma patients were found to have the lowest mOPP of $<50 \mathrm{~mm} \mathrm{Hg}(47.8 \mathrm{~mm} \mathrm{Hg})$. This group of patients was also noted to have the highest mean IOP $(16.7 \mathrm{~mm} \mathrm{Hg})$ and lowest mean SBP $(132.7 \mathrm{~mm} \mathrm{Hg})$, both of which mathematically translated into a low OPP. In advanced glaucoma eyes which are already compromised structurally and functionally, low OPP may exert a significant effect in hastening the process of 

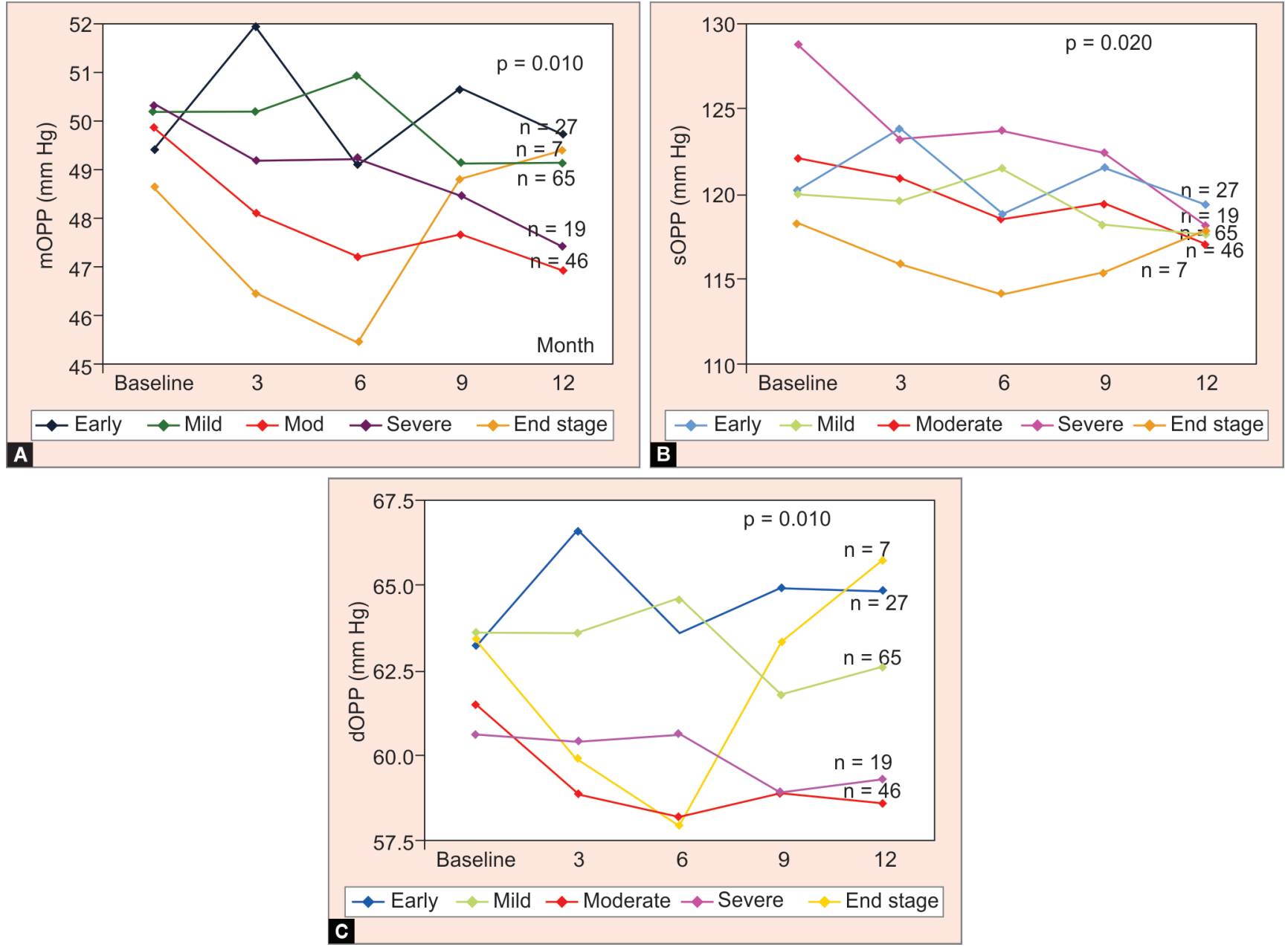

Figs $1 \mathrm{~A}$ to $\mathrm{C}$ : Mean OPP and severity of AGIS score

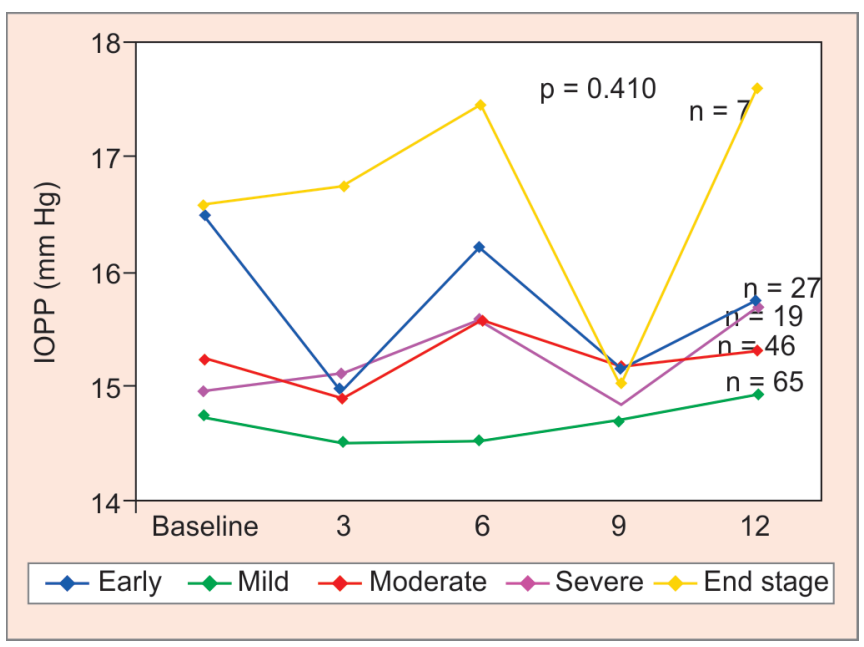

Fig. 2: Mean inter-visits IOP and severity of glaucoma

retinal fiber layer damage. ${ }^{21}$ The relationship between mOPP and different severity of glaucoma has not been explored. But OPP as a risk factor for glaucoma was previously reported with variable results. In Los Angeles Latino Eye Study, up to 3.6-folds increase risk of OAG were identified among individuals with $\mathrm{mOPP} \leq 50 \mathrm{~mm}$ $\mathrm{Hg} .{ }^{5}$ The Barbados Eye Study also found a 3.1-folds increase risk of developing glaucoma at 4 years with $\mathrm{mOPP}<42 \mathrm{~mm} \mathrm{Hg}$.

In the present study, patients with end-stage glaucoma shown the widest mOPP fluctuation $(4 \mathrm{~mm} \mathrm{Hg})$ compared with patients with early $(2.5 \mathrm{~mm} \mathrm{Hg})$ and mild $(2 \mathrm{~mm} \mathrm{Hg})$ glaucoma. Choi et al. reported that fluctuation of circadian OPP is a consistent risk factor for progression to more severe disease..$^{13}$ In their study, patients with NTG have wider circadian fluctuation and are associated with worse structural and functional outcome..$^{13}$ It is believed that vascular dysregulation within the retrobulbar hemodynamic in patients with NTG may be responsible for OPP fluctuation causing unstable ONH perfusion leading to retinal nerve fiber layer damage. ${ }^{22}$

On the other hand, the Early Manifest Glaucoma Trial (EMGT) identified that sOPP of $\leq 125 \mathrm{~mm} \mathrm{Hg}$ was associated with a higher risk of progression, and a mean sBP of $>160 \mathrm{~mm} \mathrm{Hg}$ protects against progression. ${ }^{23}$ Interestingly, in our patients, the mean sOPP was $<125 \mathrm{~mm} \mathrm{Hg}$ in all patients except for severe glaucoma at baseline. Mean sOPP was highest in patients with early glaucoma, followed by mild and lowest in patients with end-stage (121.2, 119.6, and 116.2 $\mathrm{mm} \mathrm{Hg}$, respectively). Mean sBP of $<137 \mathrm{~mm} \mathrm{Hg}$ (early $136.3 \mathrm{~mm}$ $\mathrm{Hg}$, mild $134.1 \mathrm{~mm} \mathrm{Hg}$, and end-stage $132.7 \mathrm{~mm} \mathrm{Hg}$ ) were recorded in all patients. Our findings are almost similar to the Singapore Epidemiology of Eye Diseases (SEED) study. ${ }^{23}$ The SEED study 

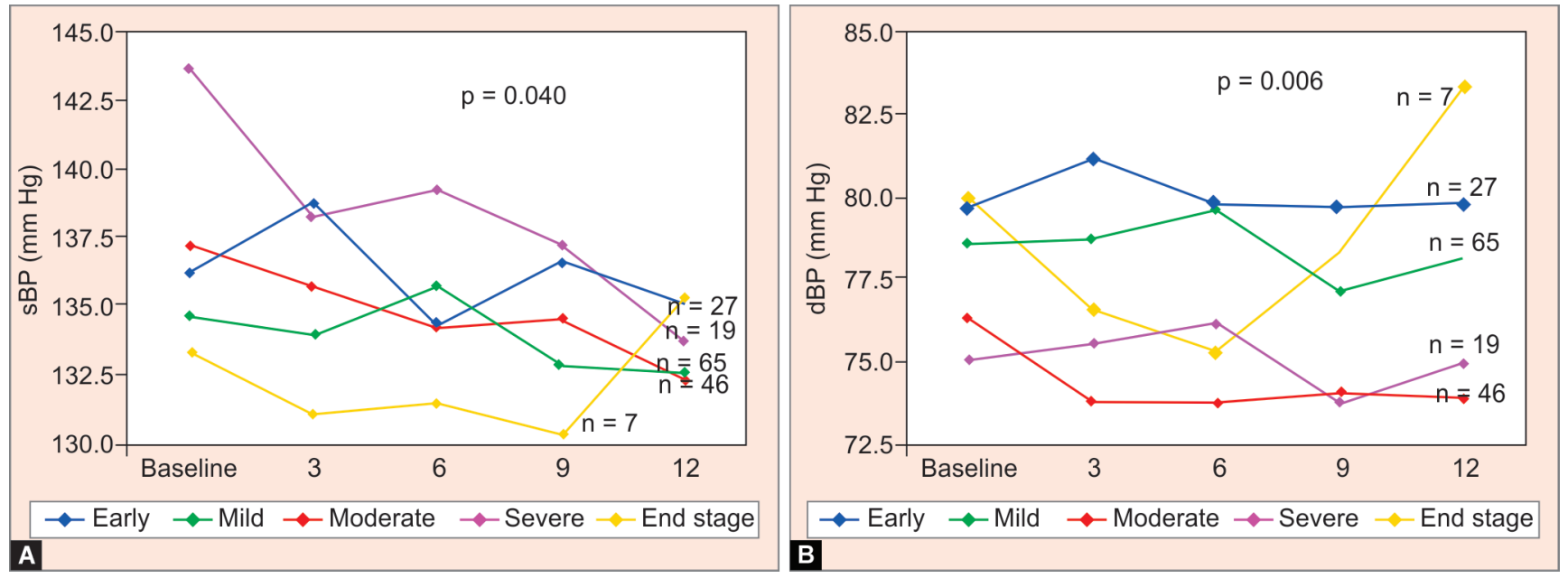

Figs 3 A and B: Mean IOP difference between follow-up visits according to severity of AGIS score. (A) Mean systolic BP according to severity of AGIS score; (B) Mean diastolic BP according to severity of AGIS score

concluded that low (<110 $\mathrm{mm} \mathrm{Hg}$ ) and high ( $>137 \mathrm{~mm} \mathrm{Hg}$ ) sOPP was significantly associated with POAG, after being adjusted to IOP. ${ }^{23}$

In our study, patients with early glaucoma, demonstrated low mean IOP $(15.6 \mathrm{~mm} \mathrm{Hg})$, higher mean dBP $(80 \mathrm{~mm} \mathrm{Hg})$, and sBP (136.5 $\mathrm{mm} \mathrm{Hg}$ ) compared with patients with end-stage glaucoma (78.7 and $132.7 \mathrm{~mm} \mathrm{Hg}$, respectively). Relatively higher BP in early glaucoma may have a protective effect on the optic nerve due to potentially high hydrostatic pressure within the small vessels providing more resistance to compression caused by IOP. ${ }^{24}$ However, as the disease progresses from early to mild or moderate glaucoma, the small vessels may start to lose their compensating ability and possibly upset the autoregulatory functions.

Based on 24-hour BP monitoring, Kaiser et al. found lower day and night time SBP at the day in patients with OAG who continued to progress despite well-controlled IOP. ${ }^{25}$ Cardiovascular disease and hypotension was found to increase the risk of rapid progression of glaucoma 2.33 times despite lower mean IOP. ${ }^{26}$ Nocturnal BP dipping and fluctuations have also been linked to the progression of glaucoma. ${ }^{27}$ Progression of glaucoma leads to a more severe stage of the disease. The AGIS reported that long-term IOP fluctuations were one of the important predictors for progression of glaucoma, even in patients with low mean IOP. ${ }^{28,29}$ Wide fluctuations could possibly cause repetitive ischemia and reperfusion insult which may have more damaging effects on the retinal nerve fiber layer compared with eyes with constant pressure.

There were limitations in our study. It was not adjusted for IOP and patients on systemic treatment for hypertension were not excluded. As we assume that BP and IOP are part of the equation in OPP, any factors affecting these two variables will theoretically affect the OPP. All patients with glaucoma were on at least one topical pressure-lowering drug. Patients with end-stage glaucoma mostly achieved their target IOP with multiple topical pressure-lowering drugs. However, we excluded those who have undergone filtering surgery. Topical pressure-lowering drugs cause reduction of IOP mathematically elevates OPP measurement. ${ }^{30}$ However, the effect of IOP on OPP is not as simple as mathematical calculation. ${ }^{27}$ Quaranta et al. reported that in their patients with glaucoma who were not on any BP-lowering medications, dOPP was significantly increased by dorzolamide $2 \%$ and latanoprost $0.005 \% .{ }^{31}$ Brimonidine $0.2 \%$, on the other hand, induced a significant decreased in dOPP but there was no significant difference with timolol $0.5 \% .{ }^{31}$ Both timolol and brimonidine were also found to significantly reduce SBP and $\mathrm{dBP}^{30,31}$ Topical pressure-lowering drugs could therefore induce changes to OPP by affecting IOP, BP, or both.

More than half of our study population were also treated for systemic hypertension. The manipulation of BP by oral antihypertensive agents can have effects not only on the mean OPP but possibly indirectly on the retinal nerve fiber layer integrity through the pressure effect. American College of Cardiology and American Heart Association in their latest report on hypertension has redefined normal BP to $120 / 80$ or below. ${ }^{32}$ Ocular perfusion pressure can be expected to be lower with the more aggressive BP treatment depending on target BP. Aggressive BP treatment could also contribute to a more frequent occurrence of hemodynamic instability (e.g., greater nocturnal BP dips) and hence ONH insult. In susceptible individuals, failure of $\mathrm{ONH}$ reperfusion to compensate for the overly pharmacologically induced low BP may occur. ${ }^{9,27}$

Hayreh found that patients on both topical and systemic betablocker medications had more occurrence of nocturnal BP drops. ${ }^{9}$ Interestingly, our patients demonstrated a downward trend of BP with each visit in all stages of glaucoma. This may be due to better compliance of patients on oral anti-hypertensive medication once they were enrolled in the study. However, office measurement of $\mathrm{BP}$ and IOP may not be the true reflection of the patient's BP and IOP. Blood pressure and IOP follow the physiological circadian rhythm and are affected by habitual position. Moreover, it is not uncommon for some patients to have "white coat hypertension" or even "reverse white coat" effect (masked hypertension), which may give rise to inaccurate $\mathrm{BP}$ interpretation. ${ }^{33}$ There is also a possibility of "regression towards the mean" that normally occurs in the study involving repeated measurements such as BP and IOP. ${ }^{34}$

In this study, three types of primary glaucoma were included; POAG, NTG, and PACG. Primary angle-closure glaucoma patients tend to have wider 24-hour IOP fluctuation and higher mean IOP than the other types. Subdividing them according to severity without looking into types of glaucoma may not reflect the actual condition as the IOP results were presented as an average mean of IOP readings. There were only seven patients with endstage glaucoma due to the high drop-out rate in this group. This happened due to their inability to provide a reliable visual field, 
and also, in the course of the study, there were inevitable changes in the management, e.g., needs for filtering surgery. The AGIS score adopted in this study may also not be ideal in determining the severity of the disease as it is based on the total deviation plot of Humphrey's visual field analysis. The effect of media opacity especially cataracts was not eliminated in this plot.

Nevertheless, balancing BP and IOP as well as minimizing intervisit OPP fluctuation is important in the management of glaucoma particularly at the early stage of glaucoma. Arresting further progression is crucial at the early stage of the disease. However, balancing BP and IOP is not as easy as mathematical calculation.

\section{Conclusion}

Our study demonstrated the importance of monitoring OPP rather than just IOP alone in the management of glaucoma especially at the early stage of the disease to prevent further progression. However, many factors are affecting OPP calculation. Balancing BP in glaucoma patients is crucial especially in those who achieved target IOP and on systemic antihypertensive treatment.

\section{References}

1. Collaborative Normal-Tension Glaucoma Study Group. The effectiveness of intraocular pressure reduction in the treatment of normal-tension glaucoma. Am J Ophthalmol 1998;126(4):498-505. DOI: 10.1016/S0002-9394(98)00272-4.

2. Bonomi L, Marchini G, Maraffa M, et al. Vascular risk factors for primary open angle glaucoma. The egna-neumarkt study. Ophthalmology 2000;107(7):1287-1293. DOI: 10.1016/s0161-6420(00)00138-x.

3. Quigley HA, West SK, Rodriguez J, et al. The prevalence of glaucoma in a population-based study of Hispanic subjects: proyecto VER. Arch Opthalmol 2001;119(12):1819-1826. DOI: 10.1001/archopht.119.12.1819.

4. Tielsch JM, Katz J, Sommer A, et al. Hypertension, perfusion pressure, and primary open-angle glaucoma: a population-based assessment. Archives Ophthalmol 1995;113(2):216. DOI: 10.1001/arc hopht.1995.01100020100038.

5. Memarzadeh F, Ying-Lai M, Chung J, et al., Los Angeles Latino Eye Study Group Blood pressure, perfusion pressure, and open-angle glaucoma: the Los Angeles Latino eye study. Invest Opthalmol Vis Sci 2010;51(6):2872-2877. DOI: 10.1167/iovs.08-2956.

6. Leske MC, Wu SY, Hennis A, et al. Risk factors for incident openangle glaucoma: the barbados eye studies. Ophthalmology 2008;115(10):85-93. DOI: 10.1016/j.ophtha.2007.03.017.

7. Harris A, Werne A, Cantor LB. Vascular abnormalities in glaucoma: from population-based studies to the clinic. Ophthalmol Clin North America 2008;145(4):595-597. DOI: 10.1016/j.ajo.2007.12.019.

8. Werne A, Harris A, Moore D, et al. The circadian variations in systemic blood pressure, ocular perfusion pressure, and ocular blood flow: risk factors for glaucoma? Surv Ophthalmol 2008;53(6):559-567. DOI: 10.1016/j.survophthal.2008.08.021.

9. Hayreh SS. Blood flow in the optic nerve head and factors that may influence it. Prog Retinal Eye Res 2001;20(5):595-624. DOI: 10.1016/ s1350-9462(01)00005-2.

10. Drance SM. Disc hemorrhages in the glaucomas. Surv Ophthalmol 1989;33(5):331-337. DOI: 10.1016/0039-6257(89)90010-6.

11. Yan DB, Coloma FM, Metheetrairut A, et al. Deformation of the lamina cribrosa by elevated intraocular pressure. $\mathrm{Br} J$ Ophthalmol 1994;78(8):643-648. DOI: 10.1136/bjo.78.8.643.

12. Fechtner RD, Weinreb RN. Mechanisms of optic nerve damage in primary open angle glaucoma. Surv Ophthalmol 1994;39(1):23-42. DOI: 10.1016/s0039-6257(05)80042-6.

13. Choi J, Kim KH, Jeong J, et al. Circadian fluctuation of mean ocular perfusion pressure is a consistent risk factor for normal-tension glaucoma. Invest Ophthalmol Vis Sci 2007;48(1):104-111. DOI: 10.1167/ iovs.06-0615.

14. Nicolela MT, Walman BE, Buckley AR, et al. Various glaucomatous optic nerve appearances. A color Doppler imaging study of retrobulbar circulation. Ophthalmology 1996;103(10):1670. DOI: 10.1016/s01616420(96)30448-x.

15. Asrani S, Zeimer R, Wilensky J, et al. Large diurnal fluctuations in intraocular pressure are an independent risk factor in patients with glaucoma. J Glaucoma 2000;9(2):134. DOI: 10.1097/00061198200004000-00002.

16. Pikilidou MI, Tsirou E, Stergiou GS, et al. Effect of hospitalization on 24-h ambulatory blood pressure of hypertensive patients. Hypertens Res 2010;33(10):995-999. DOI: 10.1038/hr.2010.127.

17. Foster PJ, Buhrmann R, Quigley HA, et al. The definition and classification of glaucoma in prevalence surveys. $\mathrm{Br} \mathrm{J}$ Ophthalmol 2002;86(2):238-242. DOI: 10.1136/bjo.86.2.238.

18. Anderson DR. Collaborative normal tension glaucoma study. Curr Opin Ophthalmol 2003;14(2):86. DOI: 10.1097/00055735-20030400000006.

19. Bild DE, Bluemke DA, Burke GL, et al. Multi-ethnic study of atherosclerosis: objectives and design. Am J Epidemiol 2002;156(9):871-881. DOI: 10.1093/aje/kwf113.

20. Sehi M, Flanagan JG, Zeng L, et al. Relative change in diurnal mean ocular perfusion pressure: a risk factor for the diagnosis of primary open-angle glaucoma. Invest Ophthalmol Vis Sci 2005;46(2):561-567. DOI: $10.1167 /$ iovs.04-1033.

21. Renard E, Palombi K, Gronfier C, etal. Twenty-four hour (nyctohemeral) rhythm of intraocular pressure and ocular perfusion pressure in normal-tension glaucoma. Invest Ophthalmol Vis Sci 2010;51(2):882889. DOI: $10.1167 /$ iovs.09-3668.

22. Galassi F, Giambene B, Varriale R. Systemic dysregulation and retrobulbar hemodynamics in normal-tension glaucoma. Invest Ophthalmol Vis Sci 2011;5(7):4467-4471. DOI: 10.1167/iovs. 10-6710.

23. Tham YC, Lim SH, Gupta P, et al. Inter-relationship between ocular perfusion pressure, blood pressure, intraocular pressure profiles and primary open-angle glaucoma: the Singapore epidemiology of eye diseases study. Br J Ophthalmol 2018;102(10):1402-1406. DOI: 10.1136/ bjophthalmol-2017-311359.

24. Riva $C E$, Hero $M$, Titze $P$, et al. Autoregulation of human optic nerve head blood flow in response to acute changes in ocular perfusion pressure. Graefe's Arch Clin Exp Ophthalmol 1997;235(10):618-626. DOI: 10.1007/BF00946937.

25. Kaiser HJ, Flammer J, Graf T, et al. Systemic blood pressure in glaucoma patients. Graefes Arch Clin Exp Ophthalmol 1993;231(12):677-680. DOI: 10.1007/BF00919280.

26. Wai Chan TC, Bala C, Siu A, et al. Risk factors for rapid glaucoma disease progression. Am J Ophthalmol 2017;180:151-157. DOI: 10.1016/ j.ajo.2017.06.003.

27. Costa VP, Harris A, Anderson D, et al. Ocular perfusion pressure in glaucoma. Acta Ophthalmol 2014;92(4):e252-e266. DOI: 10.1111/ aos.12298.

28. Nouri-Mahdavi K, Hoffman D, Coleman AL, et al. predictive factors for glaucomatous visual field progression in the advanced glaucoma intervention study. Ophthalmology 2004;111(9):1627-1635. DOI: 10.1016/j.ophtha.2004.02.017.

29. Caprioli J, Coleman AL. Intraocular pressure fluctuation a risk factor for visual field progression at low intraocular pressure in the advanced glaucoma intervention study. Ophthalmology 2008;115(7):1123-1129. DOI: 10.1016/j.ophtha.2007.10.031.

30. Harris A, Jonescu-Cuypers CP. The impact of glaucoma medication on parameters of ocular perfusion. Curr Opin Ophthalmol 2001;12(2):131137. DOI: $10.1097 / 00055735-200104000-00009$.

31. Quaranta L, Gandolfo F, Turano R, et al. Effects of topical hypotensive drugs on circadian IOP, blood pressure, and calculated ocular perfusion pressure in patients with glaucoma. Invest Ophthalmol Vis Sci 2006;47(7):2917-2923. DOI: 10.1167/iovs.05-1253. 
32. Whelton PK, Carey RM, Aronow WS, et al. 2017ACC/AHA/AAPA/ ABC/ACPM/AGS/APhA/ASH/ASPC/NMA/PCNA Guideline for the prevention, detection, evaluation, and management of high blood pressure in adults: a report of the American college of cardiology/ American heart association task force on clinical practice guidelines. J Am Coll Cardiol 2017. [Epub ahead of print].
33. Larkin KT, Schauss SL, Elnicki DM. Isolated clinic hypertension and normotension: false positives and false negatives in the assessment of hypertension. Blood Press Monit 1998;3:247-254.

34. Barnett AG, van der Pols JC, Dobson AJ. Regression to the mean: what it is and how to deal with it. Int J Epidemiol 2005;34(1):215-220. DOI: 10.1093/ije/dyh299. 